\title{
ACCRETION DISKS IN BLACK HOLE CANDIDATES OBSERVED WITH ASCA
}

\author{
T. DOTANI \\ Institute of Space and Astronautical Science \\ 3-1-1 Yoshino-dai, Sagamihara, Kanagawa 229, Japan
}

\begin{abstract}
Structure of the accretion disk is compared between the soft and hard states of Cyg X-1 using the ASCA data. Large uncertainty of the disk parameters in hard state prevent us from drawing clear conclusion, but the data are consistent with a factor of 3 larger (optically thick) inner disk boundary in the hard state than in the soft state.
\end{abstract}

\section{Introduction}

Accretion disk is believed to play an important role to power galactic and extra-galactic high energy objects. Gravitational potential energy is converted to radiation and kinematic energy through the accretion disk, but the conversion mechanisms are not fully understood yet.

Stellar mass black holes in Galaxy may be suited to investigate the accretion disk because of its proximity. We analyzed the ASCA data of Cyg X-1, which is a prototypical and the best studied black hole candidate (BHC). We compared the hard/soft state data to investigate how the disk structure changes with the state transition. Data from another BHC, LMC $\mathrm{X}-3$, are also investigated.

\section{Analysis and Results}

\subsection{SOFT STATE}

\subsubsection{Cyg $X-1$}

Cyg X-1 is mostly found in hard state, but rare transition to soft state was observed in 1996 May, which continued for about 3 months. ASCA observation of Cyg X-1 in the soft state was made in May 30-31 for net 


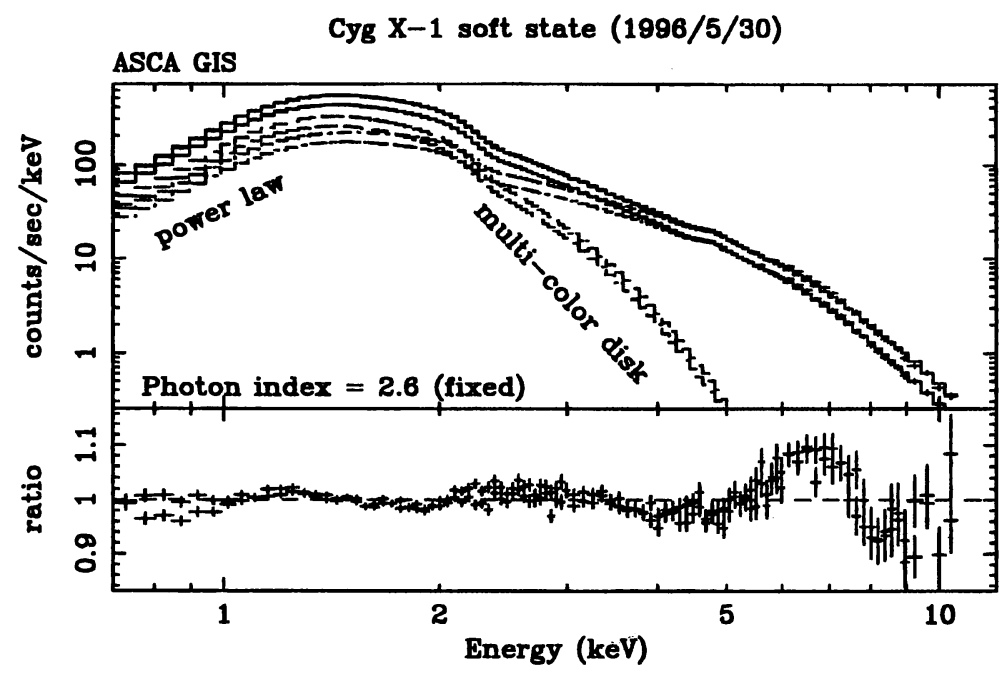

Figure 1. Energy spectrum of Cyg X-1 in the soft state obtained with ASCA GIS. Best-fit continuum model, i.e. multi-color disk model plus a power law, is also shown. Residual structure around $7 \mathrm{keV}$ may be due to a broad iron line.

exposure of $33 \mathrm{ksec}$. Spectral analysis of the data is described in Dotani et al. (1997) and Kubota et al. (1997). We just show here the energy spectrum with the model function (figure 1). The apparent temperature of the inner disk boundary was $0.43 \pm 0.01 \mathrm{keV}$ and the apparent inner disk radius to be $71_{-2}^{+13} \mathrm{~km}$ for the assumed distance of $2.5 \mathrm{kpc}$. This apparent radius corresponds to $3 R_{s}\left(R_{s}\right.$ : Schwarzschild radius) of $12 M_{\odot}$ object, which coincides with the best mass estimate of Cyg X-1. Thus, optically thick accretion disk is considered to be extended down to $3 R_{s}$ in the soft state (Balucinska-Church et al. 1997).

A power law component become prominent above $4 \mathrm{keV}$; structures are noticed around 6-7 keV as shown in the residual plot of figure 1 . The structures may be interpreted as a broad line, which is expected from highly ionized accretion disk (Cui et al. 1997).

\subsection{2. $L M C X-3$}

We observed LMC X-3, which always stay in soft state, in 1993 September and 1995 April. The energy spectra are well fitted with a power law plus a multi-color disk model. The inner disk temperature shows significant change between the two observations $(0.83 \pm 0.03 \mathrm{keV}$ in 1993 and $0.98 \pm 0.02 \mathrm{keV}$ in 1995), but the apparent disk radius stayed constant. This is just expected if an optically thick accretion disk is extended down to $3 R_{s}$. 


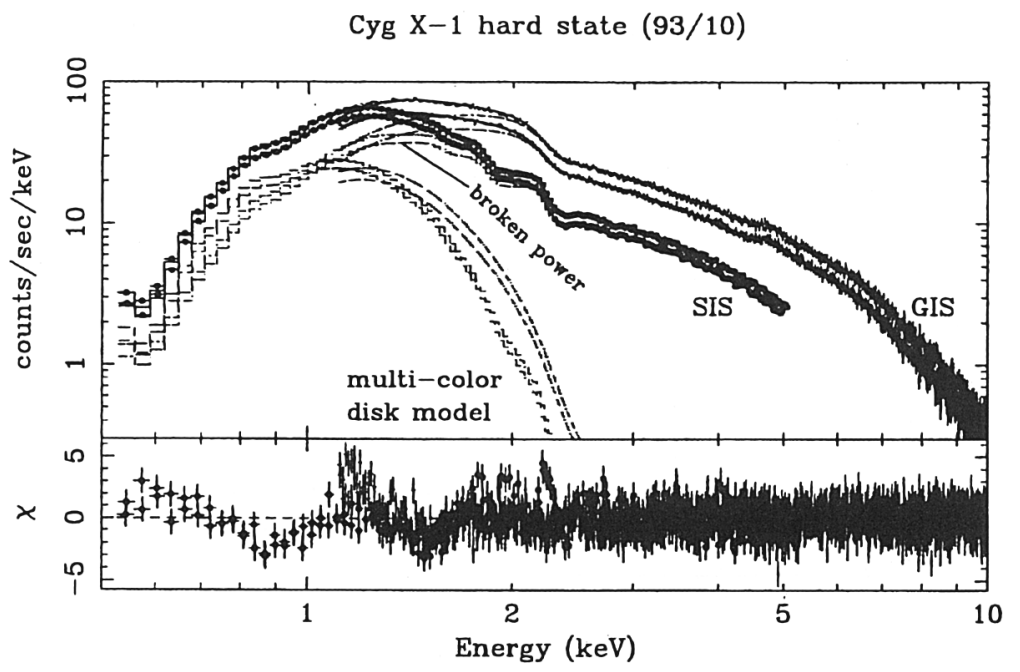

Figure 2. Energy spectrum of Cyg X-1 in the hard state obtained with ASCA. Best-fit model function, multi-color disk model plus a broken power law, is also shown.

\subsection{HARD STATE}

ASCA observed Cyg X-1 in hard state many times, because it is one of the instrumental calibration sources of the satellite. We analyzed here the data in 1993 October and November, because SIS data were available for these data. The same data were already analyzed by Ebisawa et al. (1996).

The energy spectrum and the best fit model functions are shown in figure 2 for the data in 1993 October. We used a model consisting of a multi-color disk model and a broken power law modified by the low-energy absorption and the dust scattering. We used parameters of the dust scattering halo obtained by ROSAT (Predehl and Schmitt 1995). As shown in the figure, the disk and the power law components have similar contribution in lower energy bands, and the uncertainty of the spectral shape of the power law component prevents us from determining the precise parameters of the disk emission. Allowed range of the disk parameters, in which systematic errors and time variations are also included, is shown in figure 3.

\section{Discussion}

Based on the wide band observations of Cyg X-1, Zhan et al. (1997) concluded that bolometric luminosity is almost same between hard and soft states. This means that mass accretion rate does not change during the 


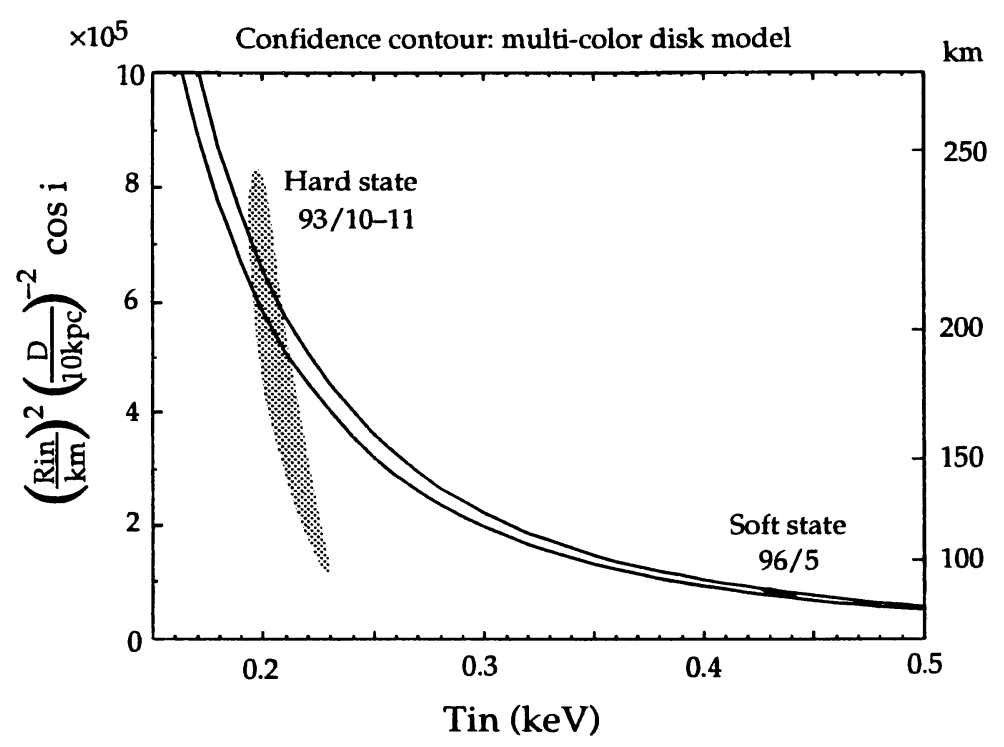

Figure 3. Allowed regions of disk parameters of Cyg X-1 determined with ASCA data. Abscissa is the color temperature at the inner disk boundary and the ordinate is a quantity proportional to the square of the inner disk radius. The regions include not only the statistical errors but also systematic errors due to the time variations and uncertainty in the power law component. See text for the solid lines.

hard/soft transition. Solid lines in figure 3 indicate the local disk temperature as a function of disk radius when the mass accretion rate is constant. As seen from the figure, hard/soft state data are consistent with only the change of inner disk radius, but the large uncertainty in the hard state data does not exclude other possibilities (Balucinska-Church et al. 1997).

Acknowledgment Present work is based on the collaboration with following people: H. Inoue, F. Nagase, K. Mitsuda, Y. Ueda, K. Asai, Y. Aruga (ISAS), K. Makishima, A. Kubota (Tokyo Univ), H. Negoro (Riken), K. Ebisawa (NASA/GSFC), W. Cui (MIT), M. Balucinska-Church, M. Church (Birmingham Univ).

\section{References}

Dotani, T. et al. 1997, ApJL, 485, L87

Cui, W. et al. 1997, this volume.

Ebisawa, K. et al. 1996, ApJ, 467, 419

Kubota, A. et al. 1997, this volume.

Balucinska-Church, M. et al. 1997, this volume.

Predehl, P. and Schmitt, J. H. M. M., 1995, A\&A, 293, 889

Zhan, S. N. et al. 1997, ApJL, 477, L95 